\title{
A review on the usage of zeolite, perlite and vermiculite as natural enhancement materials for grounding system installations
}

\begin{abstract}
Grounding system of an electrical device is most needed for the safety of power system purposes. This is due to the need to provide a low impedance medium for fault currents or electromagnetic interference signal to be dispersed into the earth as fast as possible. In the current practice, three phase power systems are grounded by connecting one or more selected neutral points to the grounding system. Therefore, the performance of the grounding system must be in a top-notch condition as it governs the efficiency of the grounding system itself. This could be done by employing enhancement materials in the vicinity of the buried earth rod of the grounding systems as it could reduce its earth resistance and maintain the low impedance value over time. There are two typical types of enhancement materials available in the market nowadays which are natural and chemical based, which are both used in order to decrease the resistivity of the surrounding soil. According to the recommendation proposed by previous researchers, the natural enhancement materials tends to be utilized more widely compared to the chemical enhancement materials due to its eco-friendly properties, corrode free earth rod in a long period of time, non-detrimental the surrounding environment and able to provide an optimum earth resistance value for the installed earth rod grounding systems. Hence, it is the main aim of this paper to highlight the advantages of using Zeolite, Perlite and Vermiculite as the natural enhancement material for grounding system purposes.
\end{abstract}

Keyword: Zeolite; Perlite; Vermiculite; Natural enhancement material; Earth resistance 